\title{
Standardization of the Korean Version of the Posttraumatic Embitterment Disorder Self-Rating Scale
}

\author{
Cheolmin Shin ${ }^{1}$, Changsu Han ${ }^{\circledR}$, Michael Linden², Jeong-Ho Chae ${ }^{3}$, Young-Hoon Ko', \\ Yong-Ku Kim¹, Seung-Hyun Kim¹, Sook-Haeng Joe' and In-Kwa Jung ${ }^{1}$ \\ ${ }^{1}$ Department of Psychiatry, Korea University College of Medicine, Seoul, Republic of Korea \\ ${ }^{2}$ Psychosomatic Rehabilitation Research Group, Charité University Medicine Berlin and the Rehabilitation Centre Seehof, Teltow/Berlin, Germany \\ ${ }^{3}$ Department of Psychiatry, College of Medicine, The Catholic University of Korea, Seoul, Republic of Korea
}

\begin{abstract}
Objective Embitterment is a persistent feeling of being let down or insulted, feeling like a "loser", or feeling revengeful but helpless. In South Korea, social injustice experienced during rapid industrial development and protracted unemployment during the Asian economic crisis may lead to strong feelings of embitterment. North Korean defectors and victims of industrial disasters may also experience humiliation and feelings of injustice. Posttraumatic Embitterment Disorder (PTED) is a recent conceptualization of a new psychiatric disorder. This study tested the reliability and validation of the Korean version of the PTED Scale.

Methods Subjects aged 18 years or older were recruited from a psychiatric outpatient clinic. All subjects were diagnosed with a depressive disorder. Subjects completed the Korean version of the PTED Scale, the Patient Health Questionnaire (PHQ-9) and the Patient Health Questionnaire (PHQ-15) at baseline and two weeks later.

Results Approximately $15.4 \%$ of subjects could be categorized as having PTED. The test-retest reliability of the PTED Scale was good $(\mathrm{r}=0.76)$ and the internal consistency was very high (Cronbach's alpha=0.962). Positive correlations were found between the PTED Scale, the PHQ-9 and the PHQ-15, indicating substantial convergent validity of the PTED Scale.

Conclusion The Korean version of the PTED Scale is a reliable and valid measurement of embitterment in Korean adults as an emotional reaction to a negative life event.

Psychiatry Investig 2012;9:368-372
\end{abstract}

Key Words Embitterment, PTED, PHQ-9, PHQ-15.

\section{INTRODUCTION}

Embitterment is a persistent feeling of being let down or insulted, feeling like a "loser", or feeling revengeful but helpless. Embitterment is often experienced as a consequence of a negative life event. For example, embitterment has been observed in east German people who had experienced sudden, uncontrollable events such as the sudden and unexpected loss of a job or uncertainty during the unification of Germany. ${ }^{1}$

In South Korea, social injustice during the rapid industrial development after the Korean war and the recent protracted

Received: May 9, 2012 Revised: June 28, 2012

Accepted: July 31, 2012 Available online: November 12, 2012

$\triangle$ Correspondence: Changsu Han, MD, PhD, MHS

Department of Psychiatry, Korea University College of Medicine, Ansan Hospital, 516 Gojan-dong, Ansan 425-707, Republic of Korea

Tel: +82-31-412-4931, Fax: +82-31-412-5144, E-mail: hancs@korea.ac.kr

(a) This is an Open Access article distributed under the terms of the Creative Commons Attribution Non-Commercial License (http://creativecommons.org/licenses/bync/3.0) which permits unrestricted non-commercial use, distribution, and reproduction in any medium, provided the original work is properly cited. unemployment during the Asian economic crisis may be causes of feelings of embitterment in Koreans. ${ }^{2}$ Additionally, North Korean defectors and victims of industrial disaster may also experience humiliation and feelings of injustice. Further, it is likely that an increased number of people may experience feelings of embitterment during the unification of South and North Korea, similar to those experienced by people during the unification of Germany. Therefore, the term 'embitterment' may be very meaningful for Korean people.

Historically, embitterment is a familiar concept for Koreans because the term "Haan (한 恨)" reflects feelings similar to the western definition of embitterment. Additionally, the Korean culture-bound syndrome, Hwa-Byung (literally means "fire disease"), is understood as an anger syndrome and has been typically viewed as a result of longstanding life stress, endurance and difficulty in expressing negative feelings within a rigid social and familial structure. ${ }^{3-6}$ Haan and Hwa-Byung may be similar to feeling embitterment and experiencing posttraumatic embitterment disorder (PTED), respectively. 
PTED is a relatively recent conceptualization of a new psychiatric disorder. Linden ${ }^{1}$ introduced the concept of PTED, which acknowledges embitterment and its possible pathological consequences. Linden ${ }^{1}$ described PTED as a distinct subgroup of adjustment disorders in which the trigger event is normal negative life event such as unemployment, divorce or death of a relative. Diagnostic criteria of PTED are: 1) development of symptoms followed by a single, exceptional, normal negative life event; 2) the event is perceived as unjust, an insult, and humiliating; 3) the event evokes feelings of embitterment, rage, and helplessness; 4) the event leads to emotional arousal in the person when reminded of the event.

Linden et al. ${ }^{7}$ created a PTED self-rating scale that is a 19item questionnaire to assess features of embittered reactions to negative life events. In this paper, authors translated the PTED Scale into Korean and tested its reliability and validity. The aim is to provide the screening instrument that helps diagnosis and treatment for this novel psychiatric disorder in Korea. Additionally, we report preliminary data on PTED prevalence and characteristics of the disease among Korean patients with depression.

\section{METHODS}

\section{Participants}

Subjects were recruited from a psychiatric outpatient clinic at a university hospital. Subjects were aged 18 years or older and were diagnosed with a depressive disorder according the Diagnostic and Statistical Manuals of Mental Disorders fourth edition, text revision (DSM-IV-TR) criteria. ${ }^{8}$ All subjects completed the Korean version of the PTED Scale at baseline and 2 weeks after baseline. Subjects also completed the PHQ-9 and PHQ-15 Patient Health Questionnaires. All subjects provided written informed consent before participation. All study procedures were reviewed and approved by the hospital institutional review board.

\section{Measurements}

\section{PTED self-rating scale}

The PTED scale is a 19-item questionnaire designed to assess the symptoms of PTED. For each item, respondents are asked to indicate to what extent the statement applies to them on the following five-point Likert scale: 0 , "not true at all"; 1 , "hardly true"; 2 , "partially true"; 3 , "very much true"; and 4 , "extremely true." In a previous report, the Cronbach's alpha of this questionnaire was reported to be 0.93 , indicating a high internal consistency. The Spearman rho correlation coefficient was 0.71 for the sum score, with a range of $0.53-0.86$ for individual items, indicating adequate test-retest reliability of the

\section{PTED Scale. ${ }^{7}$}

The original German version of the PTED Scale was translated into English by Linden and coauthors and subsequently reviewed and edited by and America English native speaker. Translation and back translation of the PTED Scale were done following state-of-the-art procedures for cross-cultural assessment. ${ }^{9}$ The translation was checked until the clinicians felt that the Korean version corresponded closely to the English version and would be meaningful to Koreans.

\section{The Patient Health Questionnaire-9 (PHQ-9)}

The nine-item version of the Patient Health Questionnaire is an instrument used for screening, diagnosing, and assessing the severity of depression. ${ }^{10-12}$ Each item is rated on a scale from 0 to 3 . The total score can range from 0 to 27 . This measure has been widely used in primary care settings for psychiatric purposes. ${ }^{13}$ The Korean version of the PHQ-9 has been translated and validated (Cronbach alpha $=0.86, \mathrm{r}=0.74){ }^{14}$

\section{The Patient Health Questionnaire (PHQ-15)}

The 15-item version of the Patient Health Questionnaire is a somatic symptom severity subscale derived from the full PHQ. ${ }^{15,16}$ It assesses 15 somatic symptoms or symptom clusters that account for more than $90 \%$ of all physical complaints reported by outpatients. ${ }^{17-19}$ Each item is rated on a scale from 0 to 2, and total scores can range from 0 to 30 . The PHQ-15 has also been translated and validated in Korean (Han et al., 2009). The Korean version of the PHQ-15 has shown good validity and reliability (Cronbach alpha $=0.87, \mathrm{r}=0.56$ ). ${ }^{20}$

\section{Statistical analysis}

Cronbach's alpha coefficient was used to assess the internal consistency of the PTED scale. To explore test-retest reliability, Pearson's correlation coefficient was calculated using 2-week assessment of PTED scores. Convergent validity of the PTED scale was verified by comparing PTED scores with scores from the PHQ-9 and PHQ-15. We calculated the Pearson's correlation coefficient to examine the relationships among the PTED, PHQ-9 and PHQ-15 scores. All analyses were conducted using the Statistical Package for the Social Sciences (SPSS) for Windows, Version 12.0 (SPSS Inc., Chicago, IL, USA).

\section{RESULTS}

A total of 194 subjects (130 females, 64 males) participated in this study. The mean (SD) age of the subjects was 48.84 (15.95) years. The mean total score of baseline measurements were $9.22 \pm 7.28$ on the PHQ-9, $8.54 \pm 6.04$ on the PHQ-15, and $27.82 \pm 19.38$ on the PTED Scale. A PTED diagnosis was assumed based on the mean total score on the PTED Scale (PT- 
ED total score divided by the number of items; cut-off score= 2.5). ${ }^{7,21}$ A total of 30 subjects (15.4\%) had a mean total PTED score greater than 2.5 points.

A total of 96 subjects (65 females, 31 males) completed the PHQ-9, PHQ-15 and the PTED Scale at two weeks. The mean total PTED score was $1.56 \pm 1.04$ at baseline and $1.49 \pm 1.03$ at two weeks. The mean PHQ-9 score at baseline was 10.47士 7.55 and at follow-up it was 9.61 \pm 7.65 . The mean PHQ-15

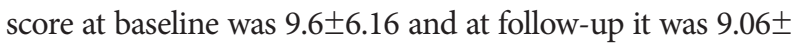
6.49. Demographic data of these subjects are presented in Table 1.

\section{Reliability}

Cronbach's alpha for the total scale was 0.97 , which reflected high internal consistency (Table 2). Pearson's correlation coefficient for inter-item correlation was 0.76 for the sum score, with a range of $0.36-0.74$ for individual items $(\mathrm{p}<0.01)$ (Table 3$)$.

\section{Validity}

The PTED total score was positively correlated with PHQ-9 and PHQ-15 scores at baseline and at two weeks. PTED scores were highly correlated with PHQ-9 score $(\mathrm{p}<0.01)$. PT-

Table 1. Demographic data

\begin{tabular}{|c|c|c|c|}
\hline & Characteristics & Frequency & Percentage \\
\hline \multirow[t]{2}{*}{ Sex } & Male & 31 & 32.3 \\
\hline & Female & 65 & 67.7 \\
\hline \multirow[t]{8}{*}{ Age } & $11-20$ & 1 & 1.0 \\
\hline & $21-30$ & 7 & 7.3 \\
\hline & $31-40$ & 23 & 24.0 \\
\hline & $41-50$ & 21 & 21.9 \\
\hline & $51-60$ & 22 & 22.9 \\
\hline & $61-70$ & 15 & 15.6 \\
\hline & $71-80$ & 6 & 6.3 \\
\hline & $80<$ & 1 & 1.0 \\
\hline \multirow[t]{7}{*}{ Education (years) } & $6>$ & 7 & 7.3 \\
\hline & 6 & 17 & 17.7 \\
\hline & 9 & 16 & 16.7 \\
\hline & 12 & 33 & 34.4 \\
\hline & 14 & 9 & 9.4 \\
\hline & 16 & 13 & 13.5 \\
\hline & $16<$ & 1 & 1.0 \\
\hline \multirow[t]{2}{*}{ Job } & Yes & 26 & 27.1 \\
\hline & No & 70 & 72.9 \\
\hline \multirow[t]{5}{*}{ Marital status } & Unmarried & 10 & 10.4 \\
\hline & Married & 74 & 77.1 \\
\hline & Divorce & 5 & 5.2 \\
\hline & Bereaved & 4 & 4.2 \\
\hline & Separated & 3 & 3.1 \\
\hline
\end{tabular}

Table 2. Internal consistency analysis of the PTED Self-rating Scale $(\mathrm{N}=194)$

\begin{tabular}{|c|c|c|}
\hline Item & $\begin{array}{l}\text { Corrected item-total } \\
\text { correlation }\end{array}$ & $\begin{array}{c}\text { Cronbach's alpha if } \\
\text { item deleted }\end{array}$ \\
\hline 1. & 0.735 & 0.965 \\
\hline 2. & 0.774 & 0.964 \\
\hline 3. & 0.686 & 0.965 \\
\hline 4. & 0.793 & 0.964 \\
\hline 5. & 0.868 & 0.963 \\
\hline 6. & 0.658 & 0.966 \\
\hline 7. & 0.812 & 0.964 \\
\hline 8. & 0.782 & 0.964 \\
\hline 9. & 0.802 & 0.964 \\
\hline 10. & 0.779 & 0.964 \\
\hline 11. & 0.715 & 0.965 \\
\hline 12. & 0.773 & 0.964 \\
\hline 13. & 0.601 & 0.966 \\
\hline 14. & 0.732 & 0.965 \\
\hline 15. & 0.835 & 0.963 \\
\hline 16. & 0.824 & 0.964 \\
\hline 17. & 0.781 & 0.964 \\
\hline 18. & 0.729 & 0.965 \\
\hline 19. & 0.775 & 0.964 \\
\hline \multicolumn{3}{|c|}{ PTED: posttraumatic embitterment disorder } \\
\hline \multicolumn{2}{|c|}{ PTED Scale items } & Coefficients \\
\hline \multicolumn{2}{|c|}{ Item 1} & $0.610^{* *}$ \\
\hline \multicolumn{2}{|c|}{ Item 2} & $0.634^{* *}$ \\
\hline \multicolumn{2}{|c|}{ Item 3} & $0.653^{* *}$ \\
\hline \multicolumn{2}{|c|}{ Item 4} & $0.546^{* *}$ \\
\hline \multicolumn{2}{|c|}{ Item 5} & $0.742^{* *}$ \\
\hline \multicolumn{2}{|c|}{ Item 6} & $0.682^{* *}$ \\
\hline \multicolumn{2}{|c|}{ Item 7} & $0.616^{* *}$ \\
\hline \multicolumn{2}{|c|}{ Item 8} & $0.598^{* *}$ \\
\hline \multicolumn{2}{|r|}{ Item 9} & $0.653^{* *}$ \\
\hline \multicolumn{2}{|r|}{ Item 10} & $0.554^{* *}$ \\
\hline \multicolumn{2}{|r|}{ Item 11} & $0.612^{* *}$ \\
\hline \multicolumn{2}{|r|}{ Item 12} & $0.657^{* *}$ \\
\hline \multicolumn{2}{|r|}{ Item 13} & $0.405^{* *}$ \\
\hline \multicolumn{2}{|r|}{ Item 14} & $0.363^{* *}$ \\
\hline \multicolumn{2}{|r|}{ Item 15} & $0.640^{* *}$ \\
\hline \multicolumn{2}{|r|}{ Item 16} & $0.610^{* *}$ \\
\hline \multicolumn{2}{|r|}{ Item 17} & $0.604^{* *}$ \\
\hline \multicolumn{2}{|r|}{ Item 18} & $0.648^{* *}$ \\
\hline \multicolumn{2}{|r|}{ Item 19} & $0.697^{* *}$ \\
\hline \multicolumn{2}{|r|}{ Sum } & $0.762^{* *}$ \\
\hline
\end{tabular}


Table 4. Correlations among PHQ-9 (1st), PHQ-15 (1st) and PTED Scale (1st)

\begin{tabular}{lccc}
\hline & PHQ-9-1st & PHQ-15-1st & PTED-1st \\
\hline PHQ-9-1st & 1 & & \\
PHQ-15-1st & $0.645^{* *}$ & 1 & \\
PTED-1st & $0.781^{* *}$ & $0.641^{* *}$ & 1 \\
\hline
\end{tabular}

${ }^{* *} \mathrm{p}<0.01$. PHQ: Patient Health Questionnaire, PTED: posttraumatic embitterment disorder

ED scores and PHQ-15 scores were also positively correlated $(\mathrm{p}<0.01)$. Correlations between the PTED, the PHQ-9 and the PHQ-15 scores are shown in Table 4.

\section{DISCUSSION}

The Korean version of the PTED Scale was shown to be stable and reliable. High internal consistency was found for the Korean version of the PTED Scale. Additionally, in this study the Korean version of the PTED Scale demonstrated good test-retest reliability. The percentage of subjects who might be diagnosed with PTED was $15.4 \%$. Previous studies have reported that the prevalence of PTED based on the PTED Scale was $2-3 \%$ among the general population and $53 \%$ among psychiatric inpatients. ${ }^{1}$ The results of the present study indicate that the prevalence of PTED in the Korean population should be validated using a formal diagnostic interview.

A high positive correlation was found between the PTED Scale and the PHQ-9. Patients with depressive disorder frequently report symptoms common to PTED (e.g., anger, unforgiveness or rumination). ${ }^{22}$ Linden et al. ${ }^{7}$ assumed in their study that many patients who were diagnosed with depressive disorder actually had PTED. However, PTED can be comorbid with depressive disorder rather than being exclusively diagnosed. Further studies are needed to evaluate the relationships between depressive disorder and PTED.

In the present study, PTED Scale scores were positively correlated with PHQ-15 scores, which implies that Korean PTED patients may report multiple somatic complaints. There are conceptual similarities between PTED and Hwa-Byung, a Korean culture-bound anger syndrome. It is possible that PTED patients in the Korean population experience somatic symptoms in common with Hwa-Byung patients. Future studies are needed to examine whether these somatic complaints are limited to Koreans.

This study has a few limitations. First, our findings may not be generalizable to people in other areas of Korea. More studies are needed to include a more representative sample. Another limitation is the fact that all participants were being treated for their depression, which may have affected their responses. Thirdly, a self rating scale was developed for screening a disorder and not for making a diagnosis. In this study, authors did not perform structured diagnostic interview or other event-related scales. Hence, we could not report the sensitivity or specificity of translated scale. Instead, we tested the convergent validity of the PTED Scale with the PHQ-9 and PHQ-15 because PTED overlap symptom profile with depressive disorders.

In conclusion, this study showed that the Korean version of the PTED Scale is a valid and reliable measure for assessing embitterment, an emotional reaction to negative life events. It is expected that the PTED Scale will be widely used in clinical settings and be helpful in uncovering characteristics of PTED in the Korean population.

\section{Acknowledgments}

This study was supported by a grant of the Korean Health Technology R\&D Project, Ministry of Health \& Welfare, Republic of Korea (A070001).

\section{REFERENCES}

1. Linden M. Posttraumatic embitterment disorder. Psychother Psychosom 2003;72:195-202.

2. Uutela A. Economic crisis and mental health. Curr Opin Psychiatry 2010;23:127-130.

3. Min SK, Suh SY. The anger syndrome hwa-byung and its comorbidity. J Affect Disord 2010;124:211-214.

4. Lin KM. Hwa-Byung: a Korean culture-bound syndrome? Am J Psychiatry 1983;140:105-107.

5. Min SK, Suh SY, Song KJ. Symptoms to use for diagnostic criteria of hwa-Byung, an anger syndrome. Psychiatry Investig 2009;6:7-12.

6. Min SK, Lee JS, Han JO. A psychiatric study on Hahn. J Korean Neuropsychiatr Assoc 1997;36:603-611.

7. Linden M, Baumann K, Lieberei B, Rotter M. The Post-Traumatic Embitterment Disorder Self-Rating Scale (PTED Scale). Clin Psychol Psychother 2009;16:139-147.

8. American Psychiatric Association. Diagnostic and Statistical Manuals of Mental disorders, Fourth Edition, text revision. Washington: American Psychiatric Association; 2000.

9. Bracken B, Barona A. State-of-the-art procedures for translating, validating, and using psychoeducational tests in crosscultural assessment. School Psychol Int 1991;12:119-132.

10. Kroenke K, Spitzer RL, Williams JB. The PHQ-9: validity of a brief depression severity measure. J Gen Intern Med 2001;16:606-613.

11. Martin A, Rief W, Klaiberg A, Braehler E. Validity of the Brief Patient Health Questionnaire Mood Scale (PHQ-9) in the general population. Gen Hosp Psychiatry 2006;28:71-77.

12. Lowe B, Unutzer J, Callahan CM, Perkins AJ, Kroenke K. Monitoring depression treatment outcomes with the patient health questionnaire-9. Med Care 2004;42:1194-1201.

13. Pinto-Meza A, Serrano-Blanco A, Penarrubia MT, Blanco E, Haro JM. Assessing depression in primary care with the PHQ-9: can it be carried out over the telephone? J Gen Intern Med 2005;20:738-742.

14. Han C, Jo SA, Kwak JH, Pae CU, Steffens D, Jo I, et al. Validation of the Patient Health Questionnaire-9 Korean version in the elderly population: the Ansan Geriatric study. Compr Psychiatry 2008;49:218-223.

15. Kroenke K, Spitzer RL, Williams JB. The PHQ-15: validity of a new measure for evaluating the severity of somatic symptoms. Psychosom Med 2002;64:258-266.

16. Kroenke K, Spitzer RL, Williams JB, Lowe B. The Patient Health Questionnaire Somatic, Anxiety, and Depressive Symptom Scales: a systematic review. Gen Hosp Psychiatry 2010;32:345-359. 
17. van Ravesteijn $\mathrm{H}$, Wittkampf $\mathrm{K}$, Lucassen $\mathrm{P}$, van de Lisdonk $\mathrm{E}$, van den Hoogen $\mathrm{H}$, van Weert $\mathrm{H}$, et al. Detecting somatoform disorders in primary care with the PHQ-15. Ann Fam Med 2009;7:232-238.

18. Aragona M, Tarsitani L, Colosimo F, Martinelli B, Raad H, Maisano B, et al. Somatization in primary care: a comparative survey of immigrants from various ethnic groups in Rome, Italy. Int J Psychiatry Med 2005; 35:241-248.

19. Interian A, Allen LA, Gara MA, Escobar JI, Diaz-Martinez AM. Somatic complaints in primary care: further examining the validity of the Patient Health Questionnaire (PHQ-15). Psychosomatics 2006;47:392-398.
20. Han C, Pae CU, Patkar AA, Masand PS, Kim KW, Joe SH, et al. Psychometric properties of the Patient Health Questionnaire-15 (PHQ-15) for measuring the somatic symptoms of psychiatric outpatients. Psychosomatics 2009;50:580-585.

21. Linden M, Rotter M, Baumann K, Lieberei B. Posttraumatic Embitterment Disorder. Cambridge: Hogrefe \& Huber; 2007.

22. Ingersoll-Dayton $\mathrm{B}$, Torges $\mathrm{C}$, Krause $\mathrm{N}$. Unforgiveness, rumination, and depressive symptoms among older adults. Aging Ment Health 2010;14:439-449. 